\title{
Mos 2 -based absorbers: whole visible spectrum coverage with high efficiency
}

Mahdieh Hashemi ( $\square$ mahdieh.hashemi@gmail.com )

Fasa University

Narges Ansari

Alzahra University

Mahsa Vazyefi

Alzahra University

Research Article

Keywords:

Posted Date: February 24th, 2022

DOI: https://doi.org/10.21203/rs.3.rs-1353097/v1

License: (c) (i) This work is licensed under a Creative Commons Attribution 4.0 International License.

Read Full License 


\title{
$\mathrm{MoS}_{2}$-based absorbers: whole visible spectrum coverage with high efficiency
}

\author{
Mahdieh Hashemi ${ }^{1, *}$, Narges Ansari ${ }^{2, *}$, and Mahsa Vazyefi ${ }^{2}$ \\ ${ }^{1}$ Department of Physics, College of Science, Fasa University, Fasa 74617-81189, Iran \\ ${ }^{2}$ Department of Physics, Faculty of Physics and Chemistry, Alzahra University, Tehran, Iran \\ *mahdieh.hashemi@gmail.com, n.ansari@alzahra.ac.ir
}

\begin{abstract}
To design highly efficient and broadband nanometer-sized absorbers based on the atomically thin transition metal dichalcogenides (TMDCs), we propose utilizing inclined gold gratings on $\mathrm{MoS}_{2}$ monolayer. In the case of gold gratings with zero inclination, coverage of the absorption spectrum in the entire visible range occurs between the values of $42 \%$ to $73 \%$. Considerable increase in the absorbed light occurs by introducing $13 \mathrm{~nm}$ inclination to the gold gratings with equal values of the grating's period and width as $60 \mathrm{~nm}$. With the application of this grating, maximum absorption of $88 \%$ is reached and the absorption bandwidth covers the entire visible spectrum with only $12 \%$ variation of the absorption value relative to this maximum (88\%). Footprints of resonant excitation of two different modes in the absorber structure are evident: the named "reflection" mode and localized surface plasmons (LSPs). Inclination of the gratings leads the LSP modes to slide toward the $\mathrm{MoS}_{2}$ and causes a remarkable increment in the absorption efficiency. An impressive absorption value of $56 \%$ in $\mathrm{MoS}_{2}$ monolayer is gained by the gold grating's inclination of $17 \mathrm{~nm}$. The designed absorber paves a new way in designing TMDC-based absorbers with extended bandwidths and higher efficiencies.
\end{abstract}

\section{Introduction}

Higher efficiencies of optoelectronic components are directly related to the amount of light which is absorbed in them. Photovoltaic cells, photodetectors, and modulators are few examples of these components which are widely used in the field of green energy production and telecommunication ${ }^{1-3}$. Apart from the absorption quantity, the absorption bandwidth is also a deterministic parameter in absorbers, specially in photovoltaic applications within the visible range ${ }^{4,5}$.

To design miniaturized absorbers and move toward lab-on-chip-size components, two-dimensional (2D) materials together with subwavelength structures on them are proper choices. Among the atomically-thin 2D materials, efficient light absorption occurs in transition metal dichalcogenides (TMDCs) with direct band gaps ${ }^{6}$. Monolayer of $\mathrm{MoS}_{2}$, the $2 \mathrm{D}$ material that we focus on it in this paper and has direct band gap in its atomically thin state, absorbs $23 \%, 6 \%$, and $7 \%$ of the incident light at wavelengths of $432 \mathrm{~nm}, 617 \mathrm{~nm}$, and $664 \mathrm{~nm}$, respectively ${ }^{7}$. Although these absorption amounts are astonishing compared to the atomic thickness of the $\mathrm{MoS}_{2}$, to design a $\mathrm{MoS}_{2}$-based absorber these values should increase. To do this, using stacks of layers in the form of photonic crystals or quasi-photonic crystals were suggested ${ }^{4,8-12}$. Although within this method broadband absorption efficiencies above $90 \%$ is reported, due to the usage of more than hundred layers with multi-stacks of $\mathrm{MoS}_{2}$, fabrication of such structure is experimentally complicated.

Utilizing metallic structures in the form of reflecting layers, gratings, and nanoparticles in the absorber structure with their ability in reflecting back the incoming light, supporting surface plasmons (SPs) ${ }^{13}$, or localized surface plasmons (LSPs) ${ }^{14}$ are proper choices to enhance light absorption in TMDC-based absorbers ${ }^{15-18}$. SPs, the collective oscillation of electrons, and the LSPs, the trapped electric or magnetic dipoles in the metallic structures, cause enhancement of the electromagnetic field in the nearfield zone which leads to augment of the absorbed light in the structure ${ }^{19-21}$. Even in the case of using uniform metallic layers in the absorber structure in which no SPs or LSPs find the chance of excitation, metallic layers can act like reflecting mirrors which increase the light path length inside the $\mathrm{MoS}_{2}$ layer and increase the light absorption ${ }^{22,23}$.

Among $\mathrm{MoS}_{2}$-based absorbers that uses metallic layers, broadband light absorption above $80 \%$ is reported in the wavelength range of $300 \mathrm{~nm}$ to $500 \mathrm{~nm}$ which doesn't cover the full visible spectrum ${ }^{24,25}$. To extend the absorber's working spectrum, in ${ }^{17}$ a $2 \mathrm{D}$ metallic grating is used on $\mathrm{MoS}_{2}$ and a photonic crystal was added beneath it. They could extend the working spectrum but at the cost of lowering the absorption efficiency to the amounts around $70 \%$ within the wavelength range of $450 \mathrm{~nm}$ to $650 \mathrm{~nm}$. As the metallic grating dimensions are set to be subwavelength, when light collides it, not only the refractive index of the metal affects the light scattering, but also the grating geometry plays an essential role. In a graphene-based photodetector study $^{26}$, it is shown that by inclining the ribbons of gold grating, the excited LSPs will move close to the graphene layer which leads to enhanced absorption in it. 
In this paper, we design an absorber which absorbs the incident light in whole visible spectrum, from $\lambda=400 \mathrm{~nm}$ to $\lambda=780 \mathrm{~nm}$, with high efficiency, maximum absorption value of $88 \%$ and not less than $77 \%$. Our proposed absorber structure consists of inclined gold gratings on $\mathrm{MoS}_{2}$ monolayer, while the $\mathrm{MoS}_{2}$ itself stands on a plane silica substrate. By investigating the origin of the excited modes in the absorber, excitation of two types of modes in the visible spectrum can be distinguished: the named "reflection" modes at shorter wavelengths and LSP modes at longer ones. The "reflection" modes mainly capture the incoming light in the gold grating's outer face, while the LSP modes excitation happens together with light absorption enhancement in the $\mathrm{MoS}_{2}$ layer.

\section{Absorber Structure}

Our proposed absorber, as it is shown in Fig. 1, is made up of a uniform $\mathrm{MoS}_{2}$ monolayer on a silica substrate with inclined gold ribbons on it. Thickness of the $\mathrm{MoS}_{2}$ layer is indicated by $t$ which is set to $0.62 \mathrm{~nm}$ throughout the paper ${ }^{6}$. The gold ribbons with height of $h$, width of $w$, and inclination of $g$ are infinitely extended along the $z-$ and repeating themselves with periodicity of $p$ in $x$-direction (Fig. 1). By sweeping the values of these four geometrical parameters, $p, h, w$, and $g$, we explore the most efficient absorber design. The incident light impinges the absorber normally in $y$-direction with its magnetic field normal to the incident plane ( $x y$-plane). With this transverse magnetic field configuration of the illuminating light relative to the incident plane, it is known as a TM-polarized light.

To calculate the absorbed light value in the designed device, along the $y$-direction the structure terminates by perfect matched layers, while in the $x$-direction periodic boundaries surround its unit cell. Complex refractive index of MoS ${ }_{2}$, gold, and silica are taken from ${ }^{7},{ }^{27}$, and $^{28}$, respectively.

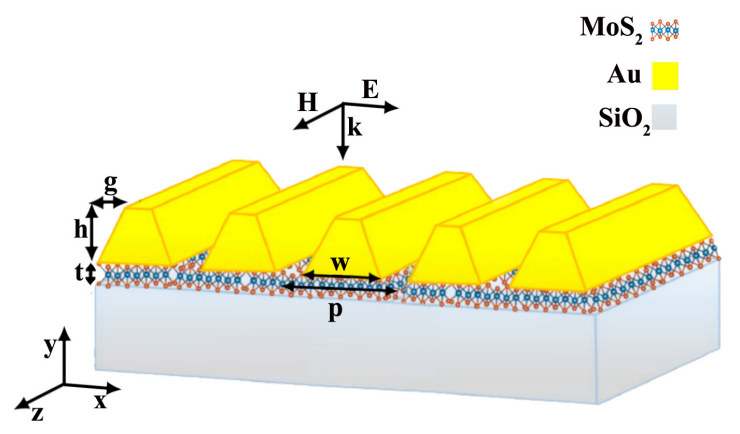

Figure 1. schematic of our designed absorber with inclined gold gratings on a $\mathrm{MoS}_{2}$ monolayer. The $p, h, w$, and $g$ parameters illustrate gold grating's periodicity, height, width and, inclination, respectively.

\section{Absorption enhancement by Non-inclined gold gratings}

In our first try, we investigate the effect of utilizing non-inclined gold grating, $g=0$, on $\mathrm{MoS}_{2}$. Absorption spectrum of $\mathrm{MoS}_{2}$ monolayer on $\mathrm{SiO}_{2}$ substrate is included in Fig. 2a as a solid black line when the only absorbing layer in the structure is $\mathrm{MoS}_{2}$. The three characteristic absorption peaks of $\mathrm{MoS}_{2}$ at the wavelengths of $432 \mathrm{~nm}, 617 \mathrm{~nm}$, and $664 \mathrm{~nm}$ which are equivalent to the energies of the direct band gaps of this material, can be clearly recognized. Utilizing gold ribbons on $\mathrm{MoS}_{2}$ layer increases both total absorption in the structure (Fig. 2a and Fig. 2c) and in the $\mathrm{MoS}_{2}$ layer (Fig. 2b and Fig. 2d). In Fig. 2a and Fig. 2b, $p$ and $h$ are set as $60 \mathrm{~nm}$ and $110 \mathrm{~nm}$, respectively, and the effect of the gold ribbon's width, $w$, on the total and MoS ${ }_{2}$ absorption is investigated. Increasing the ribbon width up to $w=50 \mathrm{~nm}$ intensifies the light absorption in a wide range of visible spectrum. If we continue increasing the ribbon's width in a way to cover fully the $\mathrm{MoS}_{2}$ as a uniform gold layer, the absorption spectrum changes to the cyan colored solid line, $w=60 \mathrm{~nm}$, of Fig. 2a and Fig. 2b. With this uniform gold layer, $w=60 \mathrm{~nm}$, as it can be seen in Fig. 2a, $60 \%$ of the incident light absorbs in the structure within the wavelength range of $\lambda=400 \mathrm{~nm}$ to $\lambda=480 \mathrm{~nm}$, while in the remaining part of the visible spectrum the absorbed light is negligible. $\mathrm{MoS}_{2}$ absorption of this structure, as it is presented in Fig. 2b, illustrates the nearly zero light absorption in $\mathrm{MoS}_{2}$ (solid cyan line). To explore the origin of this absorption, in Fig. 3a and Fig. 3b, we include the electric field distribution of the structure at $\lambda=450 \mathrm{~nm}$ with $60 \%$ light absorption and at $\lambda=760 \mathrm{~nm}$ with negligible absorption. At $\lambda=450 \mathrm{~nm}$ electric field collection at the outer surface of the gold layer shows enhanced light absorption at the gold surface in a form of a mode that we name it as "reflection". While for longer wavelengths like $760 \mathrm{~nm}$, the incident light scatters back to the free space without any absorption in the structure (Fig. 3b). 

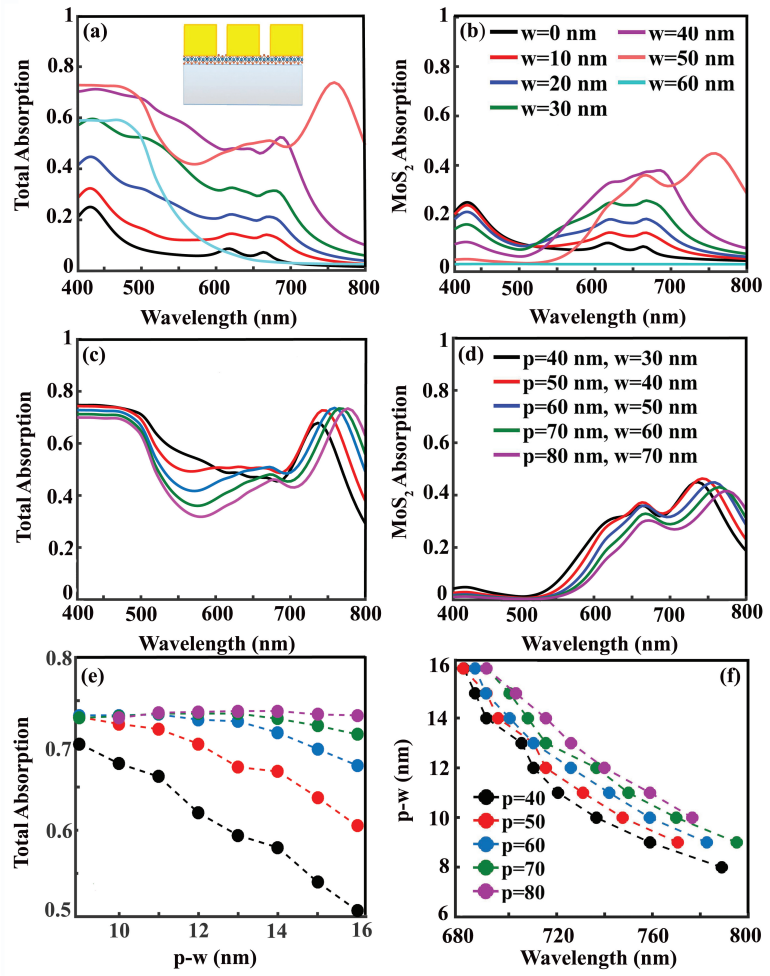

Figure 2. (a) total and (b) $\mathrm{MoS}_{2}$ absorption when the $\mathrm{MoS}_{2}$ is covered by gold grating with $p=60 \mathrm{~nm}, h=110 \mathrm{~nm}$, and width of ribbons, $w$, varies. Special cases like uniform coverage of $\mathrm{MoS}_{2}$ by gold layer ( $w=60 \mathrm{~nm}$, cyan solid line) and no gold grating ( $w=0$, black solid line) are also included. (c) total and (d) $\mathrm{MoS}_{2}$ absorption when grating pitch varies from $p=40 \mathrm{~nm}$ to $p=80 \mathrm{~nm}$ with $w$ of the gratings set for optimum absorption. (e) total absorption of the structure as a function of $p-w$ with different values of $p$ (f) $p-w$ versus wavelengths at which maximum total absorption happens for gratings with different periods.

Getting back to Fig. 2a and looking at the total light absorption in the structure with $p=60 \mathrm{~nm}$ and $w=50 \mathrm{~nm}$, with $p-w=10 \mathrm{~nm}$, the "reflection" mode excitation footprints can be distinguished with $73 \%$ light absorption between $\lambda=400 \mathrm{~nm}$ to $\lambda=480 \mathrm{~nm}$, while another resonant absorption peak at $760 \mathrm{~nm}$ is added. To investigate the nature of this enhanced absorption, in Fig. $3 \mathrm{c}$ and Fig. $3 \mathrm{~d}, H_{z}$ distribution at $\lambda=450 \mathrm{~nm}$ and $\lambda=760 \mathrm{~nm}$ are presented (as the incident light polarization is TM, the magnetic field has only $z$ component). In Fig. $3 \mathrm{c}$ and at the wavelength of $450 \mathrm{~nm}$, the same "reflection" mode as the one that is observed in Fig. 3a can be distinguished, which appears with high intensities at the outer surface of the gold grating. At $\lambda=760 \mathrm{~nm}$ resonant Fabry-Proét-type mode excitation in the grating's gap can be seen ${ }^{29,30}$. Such Fabry-Proét mode excitation which accompanies resonantly localization of the electromagnetic field in the grating's gap space is well-known as LSP. The excited LSPs couple to the incident TM polarized light in a way their magnetic field localizes at the bottom of the gap, in the vicinity of $\mathrm{MoS}_{2}$ layer, as it is clear in Fig. 3d. Field localization close to the $\mathrm{MoS}_{2}$ layer and at the bottom of the grating's gap, not only intensifies the total absorption in the structure but also enhances light absorption in $\mathrm{MoS}_{2}$ to the amount of $45 \%$ (Fig. 2b). This $45 \%$ value of the light absorption in $\mathrm{MoS}_{2}$ monolayer out of the $73 \%$ total absorption reveals the significant role of this atomically-thin layer in the absorbed light in the structure.

The occurrence of the two resonant modes, the "reflection" and the LSP modes together in the visible spectrum, prevent minimum absorption of the structure from reaching zero value in the entire range of visible wavelengths. This broadband absorption in the structure with $p=60 \mathrm{~nm}$ and $w=50 \mathrm{~nm}$ keeps the total absorption above $42 \%$ in the visible spectrum.

In Fig. 2c and Fig. 2d, gold gratings with different periods, $p$, are applied on $\mathrm{MoS}_{2}$. Width of each grating, $w$, are optimized and selected in a way to have optimum absorption. As it can be seen, increasing the grating period from $p=40 \mathrm{~nm}$ to $p=80 \mathrm{~nm}$ doesn't change the excitation range of the "reflection"-mode, $\lambda=400 \mathrm{~nm}$ to $\lambda=480 \mathrm{~nm}$, and its absorption efficiency, significantly. Within the period range of $p=50 \mathrm{~nm}$ to $p=80 \mathrm{~nm}$, the resonant LSP-based absorption peak slightly red shifts, while the peak value doesn't change significantly and fixes around $73 \%$. These fixed absorption values with grating period changes help manufacturing efficiency to be preserved against grating fabrication inaccuracy.

Excitation of LSPs in the gold grating gaps at the wavelength of the absorption peak turns our attention to the grating's 


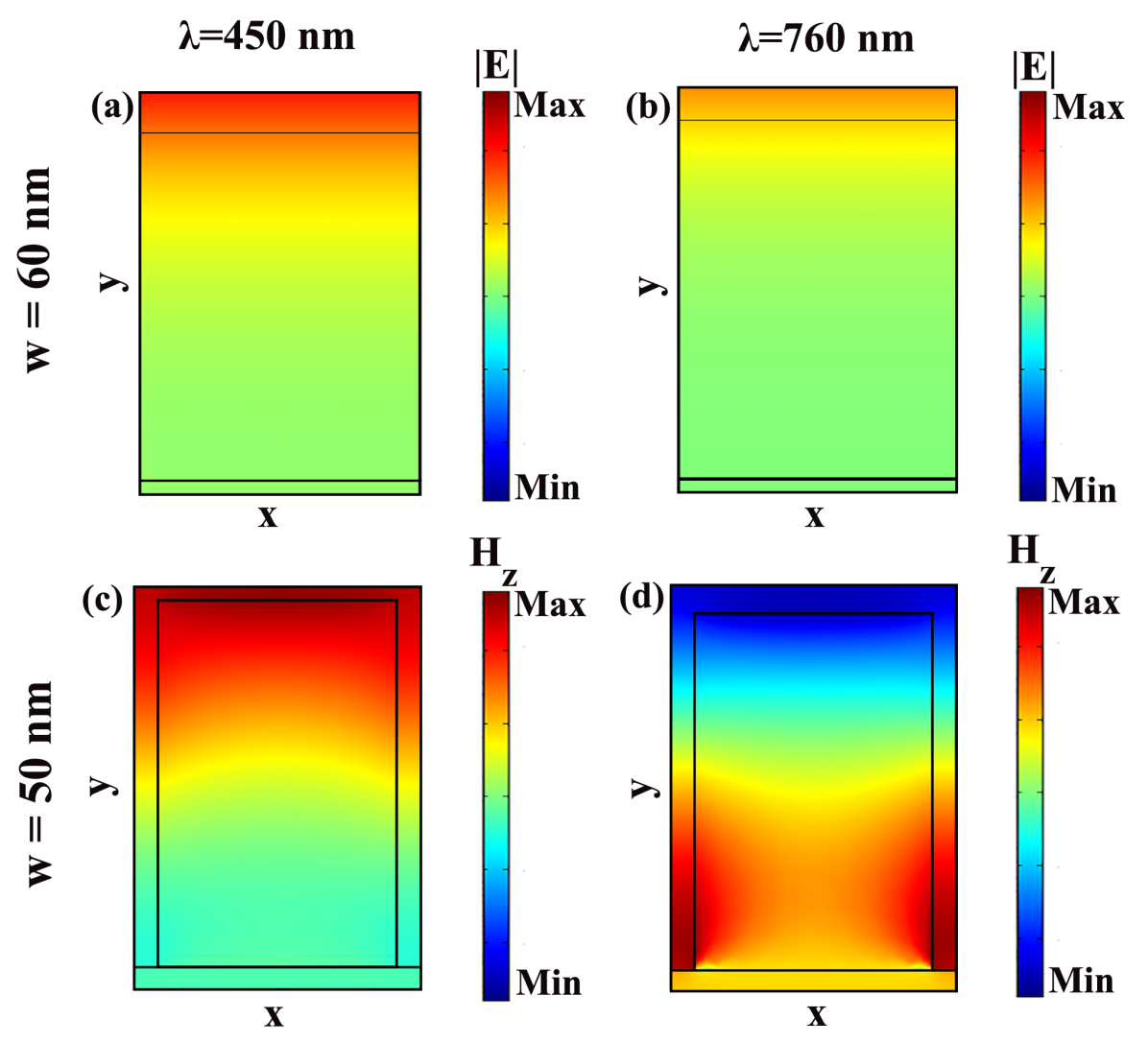

Figure 3. Electric field distribution of the structure when $110 \mathrm{~nm}$-thick uniform gold layer covers the $\mathrm{MoS}_{2}$ monolayer at (a) $\lambda=450 \mathrm{~nm}$ and (b) $\lambda=760 \mathrm{~nm}$. Electric field distribution of the structure when gold grating with $h=110 \mathrm{~nm}, p=60 \mathrm{~nm}$, and $w=50 \mathrm{~nm}$ covers the $\mathrm{MoS}_{2}$ monolayer at (c) $\lambda=450 \mathrm{~nm}$ and (d) $\lambda=760 \mathrm{~nm}$.

gap size,$p-w$, role in the absorption efficiency. In Fig. 2e, total absorption of the structure as a function of $p-w$ at the wavelength with maximum absorption is plotted for different grating periods ( $p=40 \mathrm{~nm}$ to $p=80 \mathrm{~nm}$ in steps of $10 \mathrm{~nm}$ ). With the grating's periods of 60,70 , and $80 \mathrm{~nm}$, maximum light absorption remains almost constant with nanometer-size-changes of $p-w$ from $9 \mathrm{~nm}$ to $14 \mathrm{~nm}$, while for smaller periods like $p=40 \mathrm{~nm}$ the story is totally different. Independence of the maximum light absorption from the minor changes of the grating size (up to $5 \mathrm{~nm}$ ) is a very good guarantee in experiment to get the simulated results. In a survey of the wavelength with maximum light absorption in structures with different periods as a function of the grating gap's size, $p-w$, we included the results in Fig. 2f. From Fig. 2f, it can be deduce that, maximum light absorption occurs at longer wavelengthes with smaller values of $p-w$. This way, to set the working wavelength of our absorber in the visible range, acceptable $p-w$ values should be selected between $8 \mathrm{~nm}$ to $16 \mathrm{~nm}$.

Up to now, by employing the non-inclined gold gratings on $\mathrm{MoS}_{2}$ monolayer, we reached maximum $73 \%$ of the total light absorption in the structure and $45 \%$ of $\mathrm{MoS}_{2}$ absorption, with grating parameters of $h=110 \mathrm{~nm}, p=60 \mathrm{~nm}$ to $p=80 \mathrm{~nm}$, and $p-w=9 \mathrm{~nm}$ to $p-w=16 \mathrm{~nm}$. The absorber with $p=60 \mathrm{~nm}$ in which the absorption remains above $42 \%$ in the entire visible spectrum, is the structure that we select for further studies in the next section and improving the light absorption in it while keeping its wide band absorption unaffected.

\section{Absorption enhancement by inclined gold gratings}

In a try to increase the absorption bandwidth and value simultaneously, we introduce another variation to the gold grating's structure as the ribbon's inclination. The gold grating's inclination effect is studied by sweeping over $g$ values from 0 to $25 \mathrm{~nm}$ in the grating structure with $p=60 \mathrm{~nm}$ and $w=50 \mathrm{~nm}$ that leads to the total and $\mathrm{MoS}_{2}$ absorptions presented in Fig. $4 \mathrm{a}$ and Fig. $4 \mathrm{~b}$, respectively. Compared with the absorption of the non-inclined grating, $g=0$, with the maximum total absorption of $73 \%$, the absorption reaches $80 \%$ at its maximum value with $g=5 \mathrm{~nm}$ (Fig. 4a). Interestingly, in this structure within the entire range of the visible spectrum, minimum absorption is not less than $60 \%$ which leads to a $25 \%$ variation in the total absorption 

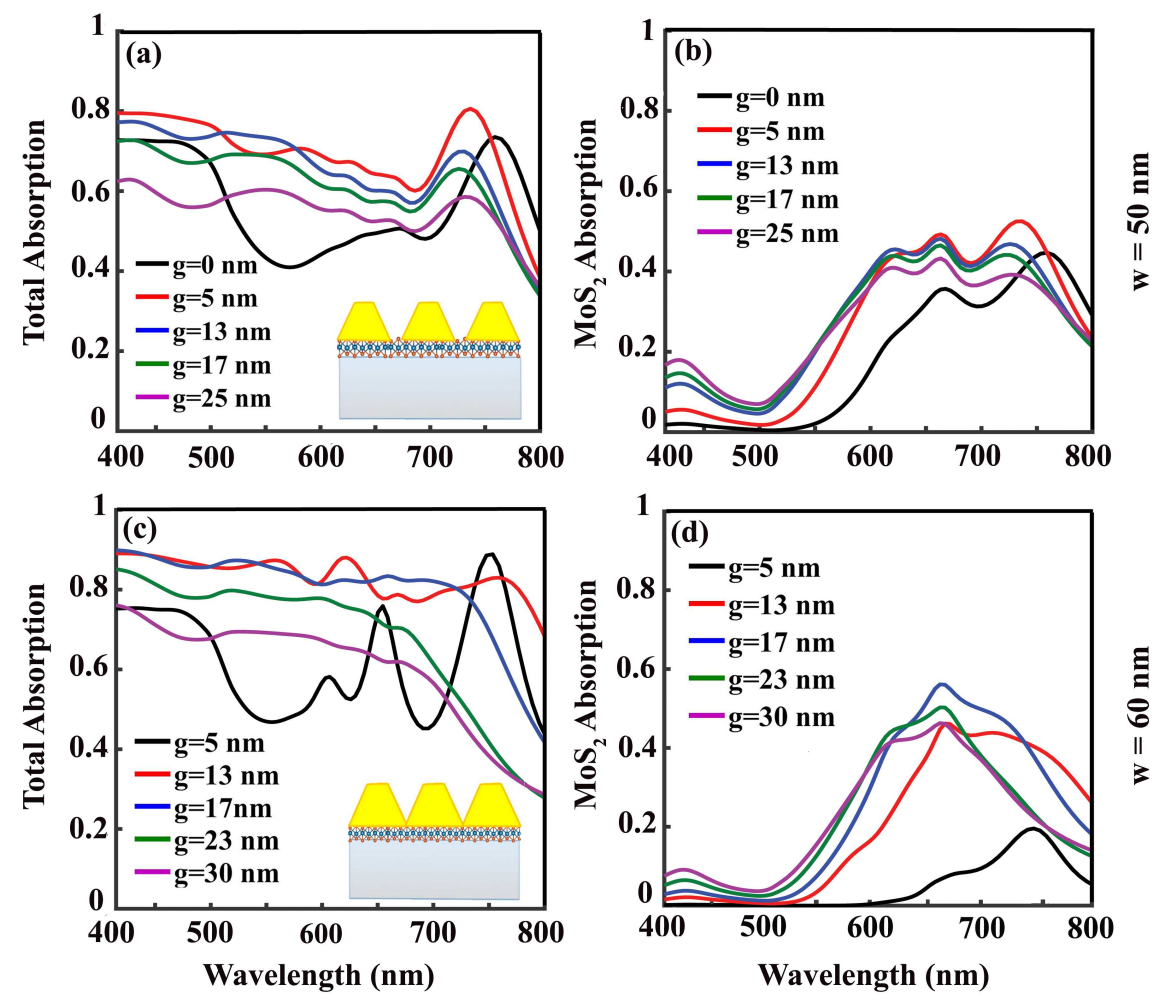

Figure 4. (a)/ (c) total and (b)/ (d) $\mathrm{MoS}_{2}$ absorption spectrum as a function of $g$, when the gold grating parameters are set to $h=110 \mathrm{~nm}, p=60 \mathrm{~nm}, w=50 \mathrm{~nm} / w=60 \mathrm{~nm}$.

relative to the maximum absorption of $80 \%$. An increase in $\mathrm{MoS}_{2}$ absorption peak value from $45 \%$ in case of $g=0$ to $52 \%$ within the structure with $g=5 \mathrm{~nm}$ is obvious in Fig. 4b. The full width at half maximum (FWHM) of the $\mathrm{MoS}_{2}$ absorption reaches $215 \mathrm{~nm}$ in case of $g=5 \mathrm{~nm}$ with this maximum absorption value of $52 \%$. This way, by introducing a slight inclination of $g=5 \mathrm{~nm}$ to the gratings, both total and $\mathrm{MoS}_{2}$ absorption values and their bandwidths increase significantly.

To continue our survey in increasing total and $\mathrm{MoS}_{2}$ absorption, we examine the case of inclined gratings with $p=w=60 \mathrm{~nm}$. It has worth of mentioning that, by considering the grating's inclination, setting the grating width equal to the grating period does not lead to a uniform gold layer extension on $\mathrm{MoS}_{2}$; instead, the gold ribbons are periodically serrated geometries as it is shown in the inset of Fig. 4c. With $g=13 \mathrm{~nm}$ not only maximum absorption reaches $88 \%$ but also surprisingly the bandwidth of the absorption spectrum extends from $\lambda=400 \mathrm{~nm}$ to $\lambda=780 \mathrm{~nm}$. In this wide wavelength range which covers almost the entire visible spectrum, one can not find any single wavelength with light absorption less than $77 \%, 12 \%$ variation relative to the maximum value, which is a great achievement (Fig. 4c). Setting $g=17 \mathrm{~nm}$ in the grating with $p=w=60 \mathrm{~nm}$ keeps the total absorption in the wavelength range of $\lambda=400 \mathrm{~nm}$ to $\lambda=720 \mathrm{~nm}$ as a smooth curve with the fixed absorption value of $81 \%$ (Fig. 4c). Maximum light absorption of $56 \%$ in $\mathrm{MoS}_{2}$ is also occurs within the structure with $\mathrm{g}=17 \mathrm{~nm}$, which is a significant value (Fig. 4d).

This way, according to the structure's application, both cases of $g=13 \mathrm{~nm}$ and $g=17 \mathrm{~nm}$ can be considered as wide-band absorbers with high efficiencies. If we were looking for a structure with efficient and wide-band absorption of $\mathrm{MoS}_{2}$, inclined gratings with $p \neq w$, e.g. the studied case of $p=60 \mathrm{~nm}$ and $w=50 \mathrm{~nm}$ with $g=5 \mathrm{~nm}$ and absorption FWHM of $215 \mathrm{~nm}$, would be more practical (Fig. 4b).

Among the studied structures, we concentrate on $p=w=60 \mathrm{~nm}$ with two cases of $g=5 \mathrm{~nm}$ in which the individual absorption peaks are well-separated and $g=13 \mathrm{~nm}$ with high absorption values in the entire range of visible spectrum and investigate the physics behind these absorptions in details. In Fig. 5a we start our study with the case of $g=5 \mathrm{~nm}$ with including the absorption (black line), reflection (red line), and transmission (blue line) of the structure. Three wavelengths are selected for detailed examination: $\lambda=450 \mathrm{~nm}$ with absorption of $75 \%$ is depicted from a wide range absorption, which extends from $\lambda=400 \mathrm{~nm}$ to $\lambda=470 \mathrm{~nm}$, and two absorption peak wavelengths $\lambda=650 \mathrm{~nm}$ and $\lambda=750 \mathrm{~nm}$ with $76 \%$ and $89 \%$ absorptions, respectively. It can be seen that, at the wavelengths with maximum light absorption, reflection dips can 

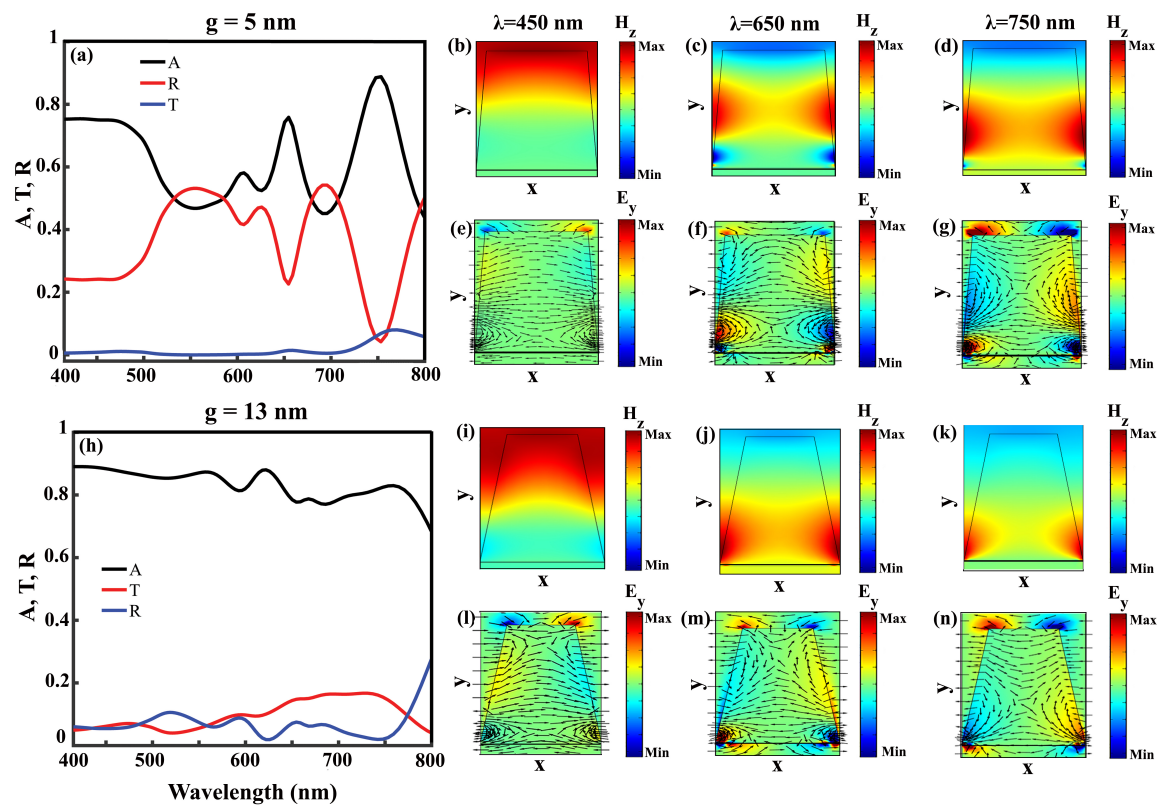

Figure 5. Reflection (R), transmission (T), and absorption (A) spectrum of the structure with $p=w=60 \mathrm{~nm}$, (a) $g=5 \mathrm{~nm}$ and (h) $g=13 \mathrm{~nm}$. At $\lambda=450 \mathrm{~nm} / \lambda=650 \mathrm{~nm} / \lambda=750 \mathrm{~nm}$, (b) and (i) $/(\mathrm{c})$ and (j) $/$ (d) and (k) show the $H_{z}$, and (e) and (l)/ (f) and $(\mathrm{m}) /(\mathrm{g})$ and $(\mathrm{n})$ illustrate the $E_{y}$ field distributions in the structure with $g=5 \mathrm{~nm}$ and $g=13 \mathrm{~nm}$, respectively.

be recognized in Fig. 5a. With the TM polarization of the incident light, the interesting $H-$ and $E$-field components for further studies are $H_{z}$ and $E_{y}$. Looking at the $H_{z}$ distribution at $\lambda=450 \mathrm{~nm}$ in Fig. $5 \mathrm{~b}$ with its related $E_{y}$ at Fig. 5e, reminds us the field distribution of the structure with $p=60 \mathrm{~nm}, w=50 \mathrm{~nm}$, with no grating inclination, $g=0$ (Fig. 3c). This way, excitation of "reflection" modes are responsible for the observed high absorption values at the wavelength range of $\lambda=400 \mathrm{~nm}$ to $\lambda=470 \mathrm{~nm}$.

At $\lambda=650 \mathrm{~nm}$, Fig. $5 \mathrm{c}$, and $\lambda=750 \mathrm{~nm}$,Fig. $5 \mathrm{~d}$, the $H_{z}$ distributions are illustrative for LSP excitation, similar to Fig. 3d, with the trapped light in the grating gaps. To find the source of this light trap, looking at the $E_{y}$ distribution is informative (Fig. $5 \mathrm{f}$ and Fig. $5 \mathrm{~g}$ ). For the ease of understanding, both arrow type and colored distribution of $E_{y}$ are included. In both Fig. $5 \mathrm{f}$ and Fig. 5g, the vortex-like $E_{y}$ arrows, together with the blue (negative minimum value) to red (positive maximum value) color changes in $E_{y}$ from one sharp edge to the other proves the resonant excitation of LSPs in the form of magnetic dipoles.

At this point, by looking at the absorption, reflection, transmission, and also $E_{y}$ and $H_{z}$ field distributions of our studied wide-band absorber with $p=w=60 \mathrm{~nm}$ and $g=13 \mathrm{~nm}$ in Fig. $5 \mathrm{~h}$ to Fig. $5 \mathrm{n}$, excitation of similar resonant modes to the studied case of $g=5 \mathrm{~nm}$ can be recognized. The studied $H_{z}$ and $E_{y}$ field distributions are included in Fig. $5 \mathrm{i}$ and Fig. 51 at $\lambda=450 \mathrm{~nm}$, Fig. 5 j and Fig. $5 \mathrm{~m}$ at $\lambda=650 \mathrm{~nm}$, and Fig. $5 \mathrm{k}$ and Fig. $5 \mathrm{n}$ at $\lambda=750 \mathrm{~nm}$, respectively. Compared to the case of $g=5 \mathrm{~nm}$, stronger localization and light trap in the grating gaps occur in the case of $g=13 \mathrm{~nm}$, which can be seen by comparing Fig. $5 \mathrm{j}$ and Fig. 5k with $g=13 \mathrm{~nm}$ with Fig. $5 \mathrm{c}$ and Fig. $5 \mathrm{~d}$ with $g=5 \mathrm{~nm}$. This stronger light localization happens together with guiding the excited LSPs close to the $\mathrm{MoS}_{2}$ layer. Taking a look at Fig. $4 \mathrm{~d}$ and comparing the $\mathrm{MoS}_{2}$ absorption of the two cases of $g=5 \mathrm{~nm}$ with maximum absorption of $20 \%$ and $g=13 \mathrm{~nm}$ with maximum $46 \%$, confirms well the accumulation of the incoming energy close to the $\mathrm{MoS}_{2}$ layer in case of $g=13 \mathrm{~nm}$. By reminding the thickness of $\mathrm{MoS}_{2}$ monolayer, $0.62 \mathrm{~nm}$, this amount of light absorption in it is surprising. Thanks to the excitation of LSPs which prepared the proper condition to use the potential of this material in our designed absorber. Height of the gold gratings, $h$, is another deterministic parameter in the absorption value which is investigated in Fig. 6. In a grating structure with $p=w=60 \mathrm{~nm}$ with $g=17 \mathrm{~nm}$, starting from $h=90 \mathrm{~nm}$ and increasing it with steps of $10 \mathrm{~nm}$ to $h=140 \mathrm{~nm}$ shows that further height increment from $h=110 \mathrm{~nm}$ doesn't change total (Fig. 6a) and $\mathrm{MoS}_{2}$ (Fig. 6b) absorptions. This way, there would be no point in increasing the grating height more than $110 \mathrm{~nm}$. Further investigations, not presented in here, show that irrespective to the value of $p$, within the structures with $p=w$, both absorption value and bandwidth reach their optimum values when $h=110 \mathrm{~nm}$, and $g=13 \mathrm{or} 17 \mathrm{~nm}$.

In Fig. 6c and Fig. 6d total and $\mathrm{MoS}_{2}$ absorptions of structures with different periods, $p$, are studied when $w$ is set to be equal to $p$ and $h=110 \mathrm{~nm}$ for two different values of $g$ as $g=13 \mathrm{~nm}$ (dotted lines) and $g=17 \mathrm{~nm}$ (solid lines). It can be deduced that increasing the grating's $p$ more than $60 \mathrm{~nm}$ doesn't change the absorption value and bandwidth significantly. It is 

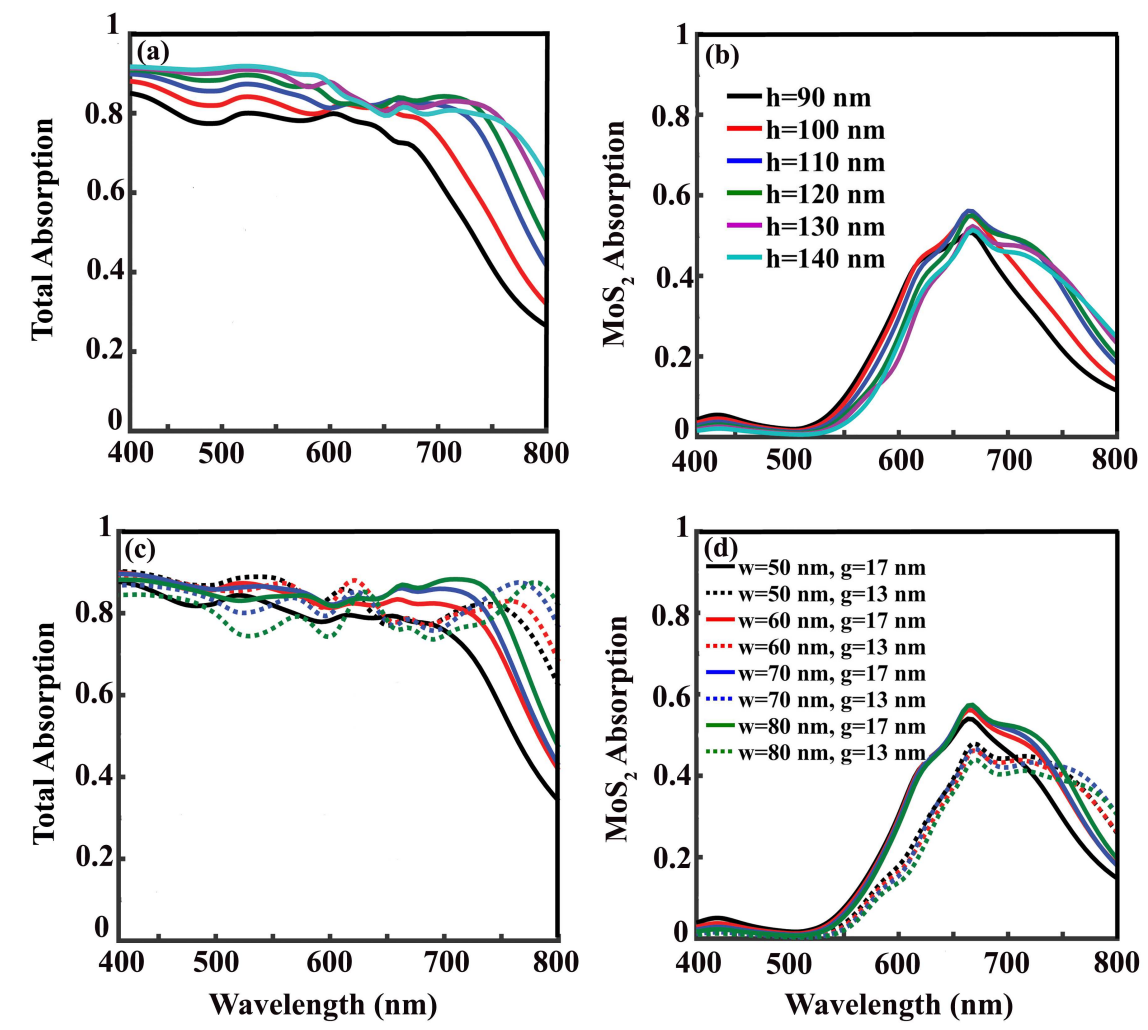

Figure 6. (a) total and (b) $\mathrm{MoS}_{2}$ absorption spectrum as a function of grating height, h, when $p=w=60 \mathrm{~nm}$ and $g=17 \mathrm{~nm}$. (c) total and (d) $\mathrm{MoS}_{2}$ absorption spectrum as a function of $p$ for two different values of $g$ as $g=13 \mathrm{~nm}$ and $g=17 \mathrm{~nm}$ with $h=110 \mathrm{~nm}$ and $w=p$.

of course a relief in designing such an absorber that if the period accuracy violates, it will not affect the absorber functionality too much. In the studied cases, with $g=13 \mathrm{~nm}$, the bandwidth of the total absorption with values above $77 \%$ is wider (up to $\lambda=780 \mathrm{~nm}$ in case of $p=60 \mathrm{~nm}$ ), while with $g=17 \mathrm{~nm}$ the bandwidth is less (up to $\lambda=720 \mathrm{~nm}$ in case of $p=60 \mathrm{~nm}$ ) but with higher values of absorption of $81 \%$. MoS 2 absorption of $56 \%$ is the maximum value that reached in gratings with $g=17 \mathrm{~nm}$ and $p$ values of 60,70 , and $80 \mathrm{~nm}$.

\section{Conclusion}

Within the design that is proposed in this paper, broadband absorption of $\mathrm{MoS}_{2}$-based absorber that covers the entire visible spectrum, from $400 \mathrm{~nm}$ to $780 \mathrm{~nm}$, with absorption efficiencies above $77 \%$ and maximum of $88 \%$ is presented. Within the shorter wavelengths, $400 \mathrm{~nm}$ to $470 \mathrm{~nm}$, the incident light mainly absorbs in the form of named "reflection" mode inside the gold grating, while in the longer wavelengths $\mathrm{MoS}_{2}$ absorption plays an essential role. Enhanced light absorption in $\mathrm{MoS}_{2}$ occurs by guiding the excited localized surface plasmons (LSPs) in the empty space between two subsequent ribbon of the gold grating toward the $\mathrm{MoS}_{2}$. Guiding the LSPs close to the $\mathrm{MoS}_{2}$ is done by inclining the gold gratings. By introducing an inclination value of $g=17 \mathrm{~nm}$ to the gratings with $p=w=60 \mathrm{~nm}, 56 \%$ of the incoming light absorbs in $\mathrm{MoS}_{2}$ monolayer. The presented method of using TMDCs with metallic structures in designing absorbers is a promising way to achieve miniaturized broadband and high efficient absorbers which are applicable in designing photovoltaic cells, photodetectors, and modulators.

\section{Data availability}

All data needed to evaluate the conclusions in the paper are present in the paper. Additional data related to this paper may be requested from the authors. 


\section{References}

1. Lee, S.-J. et al. Approach to transparent photovoltaics based on wide band gap sb2s 3 absorber layers and optics-based device optimization. ACS Appl. Energy Mater. 3, 12644-12651, DOI: 10.1021/acsaem.0c02552 (2020).

2. Hu, Q. et al. Ultra-wideband self-powered photodetector based on suspended reduced graphene oxide with asymmetric metal contacts. RSC Adv. 11, 19482-19491, DOI: 10.1039/D1RA03438C (2021).

3. Kim, S.-J. et al. Dynamic phase-change metafilm absorber for strong designer modulation of visible light. Nanophotonics 10, 713-725, DOI: doi:10.1515/nanoph-2020-0264 (2021).

4. Sen, A., Priyam, A. G., Rahman, S., Basak, A. K. \& Safayat-Al-Imam. Performance analysis of high broadband optical absorption by mose 2 monolayer in octonacci quasi-photonic crystal. In SoutheastCon 2021, 1-5, DOI: 10.1109/ SoutheastCon45413.2021.9401930 (2021).

5. Lu, Y., Yang, G., Wang, F. \& Lu, N. Enhanced photoresponse of monolayer molybdenum disulfide (mos2) based on microcavity structure. Superlattices Microstruct. 117, 163-168, DOI: https://doi.org/10.1016/j.spmi.2018.03.025 (2018).

6. Li, Y. et al. Measurement of the optical dielectric function of monolayer transition-metal dichalcogenides: $\operatorname{mos}_{2}, \mathrm{MoSe}_{2}$, $\mathrm{ws}_{2}$, and $\mathrm{WSe}_{2}$. Phys. Rev. B 90, 205422, DOI: 10.1103/PhysRevB.90.205422 (2014).

7. Ansari, N. \& Ghorbani, F. Light absorption optimization in two-dimensional transition metal dichalcogenide van der waals heterostructures. J. Opt. Soc. Am. B 35, 1179-1185, DOI: 10.1364/JOSAB.35.001179 (2018).

8. Ansari, N., Mohebbi, E. \& Fallah, K. Ultra-broadband and broad-angle absorbers with transition metal dichalcogenide monolayer using thue-morse quasi-photonic crystals. Opt. Mater. 107, 110039, DOI: https://doi.org/10.1016/j.optmat. 2020.110039 (2020).

9. Ansari, N. \& Mohebbi, E. Increasing optical absorption in one-dimensional photonic crystals including mos2 monolayer for photovoltaics applications. Opt. Mater. 62, 152-158, DOI: https://doi.org/10.1016/j.optmat.2016.09.040 (2016).

10. Ansari, N. \& Mohebbi, E. Broadband and high absorption in fibonacci photonic crystal including MoS2 monolayer in the visible range. 51, 115101, DOI: 10.1088/1361-6463/aaacbd (2018).

11. Priyam, A. G., Rahman, S., Sen, A., Basak, A. K. \& Safayat-Al-Imam. Broadband and broad-angle optical absorption by mos $<$ inf $>2</$ inf $>$ and mose $<$ inf $>2</$ inf $>$ monolayers in 1d photonic crystals. In 2021 International Symposium on Devices, Circuits and Systems (ISDCS), 1-5, DOI: 10.1109/ISDCS52006.2021.9397909 (2021).

12. Mukherjee, B. \& Simsek, E. Utilization of monolayer mos 2 in bragg stacks and metamaterial structures as broadband absorbers. Opt. Commun. 369, 89-93, DOI: https://doi.org/10.1016/j.optcom.2016.02.038 (2016).

13. Maier, S. A. Surface Plasmon Polaritons at Metal / Insulator Interfaces, 21-37 (Springer US, New York, NY, 2007).

14. Maier, S. A. Localized Surface Plasmons, 65-88 (Springer US, New York, NY, 2007).

15. Jiang, X. et al. Approaching perfect absorption of monolayer molybdenum disulfide at visible wavelengths using critical coupling. 29, 335205, DOI: 10.1088/1361-6528/aac8f1 (2018).

16. Li, H. et al. Total absorption of light in monolayer transition-metal dichalcogenides by critical coupling. Opt. Express 25, 31612-31621, DOI: 10.1364/OE.25.031612 (2017).

17. Cao, J. et al. Enhancement of broad-band light absorption in monolayer mos 2 using ag grating hybrid with distributed bragg reflector. Superlattices Microstruct. 110, 26-30, DOI: https://doi.org/10.1016/j.spmi.2017.09.008 (2017).

18. Cai, Z. et al. Sandwich-structured fe2o3@sio2@au nanoparticles with magnetoplasmonic responses. J. Mater. Chem. C 3, 11645-11652, DOI: 10.1039/C5TC01259G (2015).

19. Gong, M., Alamri, M., Ewing, D., Sadeghi, S. M. \& Wu, J. Z. Localized surface plasmon resonance enhanced light absorption in aucu/cspbcl3 core/shell nanocrystals. Adv. Mater. 32, 2002163, DOI: https://doi.org/10.1002/adma.202002163 (2020).

20. Rohizat, N. S., Ripain, A. H. A., Lim, C. S., Tan, C. L. \& Zakaria, R. Plasmon-enhanced reduced graphene oxide photodetector with monometallic of au and ag nanoparticles at vis-nir region. Sci. Reports 11, 19688, DOI: 10.1038/ s41598-021-99189-w (2021).

21. Yu, H., Peng, Y., Yang, Y. \& Li, Z.-Y. Plasmon-enhanced light-matter interactions and applications. npj Comput. Mater. 5, 45, DOI: 10.1038/s41524-019-0184-1 (2019).

22. Kaliteevski, M. et al. Tamm plasmon-polaritons: Possible electromagnetic states at the interface of a metal and a dielectric bragg mirror. Phys. Rev. B 76, 165415, DOI: 10.1103/PhysRevB.76.165415 (2007). 
23. Maurya, J. B., François, A. \& Prajapati, Y. K. Two-dimensional layered nanomaterial-based one-dimensional photonic crystal refractive index sensor. Sensors 18, DOI: 10.3390/s18030857 (2018).

24. Ansari, N., Mohebbi, E. \& Gholami, F. Enhancement of light absorption in a ws 2 monolayer using spacer and au layers. $J$. Appl. Phys. 127, 063101, DOI: 10.1063/1.5131699 (2020).

25. Ansari, N., Mohebbi, E. \& Gholami, F. Nearly perfect and broadband optical absorption by tmdcs in cover/tmdc/spacer/au/substrate multilayers. Appl. Phys. B 126, DOI: https://doi.org/10.1007/s00340-019-7352-3 (2020).

26. Hashemi, M., Farzad, M. H., Mortensen, N. A. \& Xiao, S. Enhanced absorption of graphene in the visible region by use of plasmonic nanostructures. 15, 055003, DOI: 10.1088/2040-8978/15/5/055003 (2013).

27. Johnson, P. B. \& Christy, R. W. Optical constants of the noble metals. Phys. Rev. B 6, 4370-4379, DOI: 10.1103/PhysRevB. 6.4370 (1972).

28. Ghosh, G. Dispersion-equation coefficients for the refractive index and birefringence of calcite and quartz crystals. Opt. Commun. 163, 95-102, DOI: https://doi.org/10.1016/S0030-4018(99)00091-7 (1999).

29. Hashemi, M., Farzad, M. H., Mortensen, N. A. \& Xiao, S. Enhanced plasmonic light absorption for silicon schottky-barrier photodetectors. 8, 1059-1064, DOI: 10.1007/s11468-013-9509-y (2013).

30. Søndergaard, T. \& Bozhevolnyi, S. I. Surface-plasmon polariton resonances in triangular-groove metal gratings. Phys. Rev. $B$ 80, 195407, DOI: 10.1103/PhysRevB.80.195407 (2009).

\section{Author contributions statement}

M.H. wrote the manuscript and supervised the simulations, N.A. analyzed the simulation results, and M.V. did the simulations. All authors reviewed the manuscript. 